\title{
Erratum: Light in the beam dump. Axion-Like Particle production from decay photons in proton beam-dumps
}

\author{
Babette Döbrich, ${ }^{a}$ Joerg Jaeckel ${ }^{b}$ and Tommaso Spadaro ${ }^{c}$ \\ ${ }^{a} C E R N$, \\ Esplanade des Particules 1, 1211 Geneva 23, Switzerland \\ ${ }^{b}$ Institut für theoretische Physik, Universität Heidelberg, \\ Philosophenweg 16, 69120 Heidelberg, Germany \\ ${ }^{c}$ Laboratori Nazionali di Frascati INFN, \\ Via E. Fermi 40, 00044 Frascati, Italy \\ E-mail: babette.dobrich@cern.ch, jjaeckel@thphys.uni-heidelberg.de, \\ tommaso.spadaro@cern.ch
}

ERRATUM TO: JHEP05(2019)213

ABSTRACT: We correct a number of figures in [1], in which the PYTHIA MC result of secondary $\pi^{0}$ mesons was displayed wrongly due to a missing factor of 2 . Scrutinizing the corrected data-MC comparsion, we review and model the trigger condition used in the LEBC-EHS experiment, and present an improved data-MC comparison. We review the sensitivity estimates for ALP searches in past and present experiments based on MC distributions scaled to data and find that the main conclusions of the paper remain unchanged.

ArXiv EPrint: 1904.02091 



Figure 1. Data-MC comparison of the differential cross-section of neutral pions and eta mesons produced from $400 \mathrm{GeV}(250 \mathrm{GeV})$ protons, to replace figure 3 (the right panel of figure 6) in JHEP 05 (2019) 213.
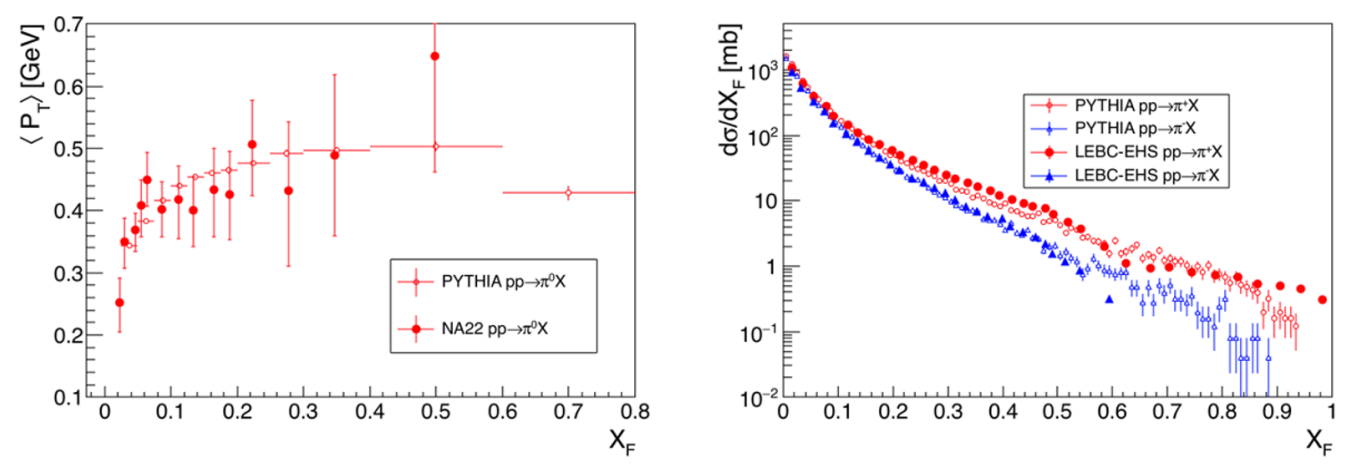

Figure 2. Left: data-MC comparison for the average transverse momentum as a function of the Feynan $X_{F}$ for a beam momentum of $250 \mathrm{GeV}$, to replace figure 7 in JHEP 05 (2019) 213. Right: data-MC comparison of the differential cross-section of charged pions produced from $400 \mathrm{GeV}$ protons.

In [1], the differential cross section for inclusive $\pi^{0}$ production in proton-proton interactions is shown as a function the Feynman variable $X_{F}=P_{Z} / P_{Z}(\max ) \sim 2 P_{Z}^{*} / \sqrt{s}$. The evaluation of $X_{F}$ after PYTHIA MC simulation was lacking a factor 2, affecting the display of the results for 250 and $400 \mathrm{GeV}$ beam energy, in the right panel of figure 6 and in figure 3 of [1], respectively. After correcting for this mistake, the new distributions are shown in figure 1. Contrary to the distribution shown in [1] the MC now populates $X_{F}$ up to large values and a segmentation of the variable at high $X_{F}$ to quantify the data-vs-MC agreement as done in table 1 of [1] seems inappropriate. The agreement with the data is better for $250 \mathrm{GeV}$ protons from the NA22 experiment [2] than for $400 \mathrm{GeV}$ protons from the LEBC-EHS experiment [3]. The correlation between average transverse momentum and $X_{F}$ in the left panel of figure 2 shows a good agreement between data and MC at $250 \mathrm{GeV}$, too. 

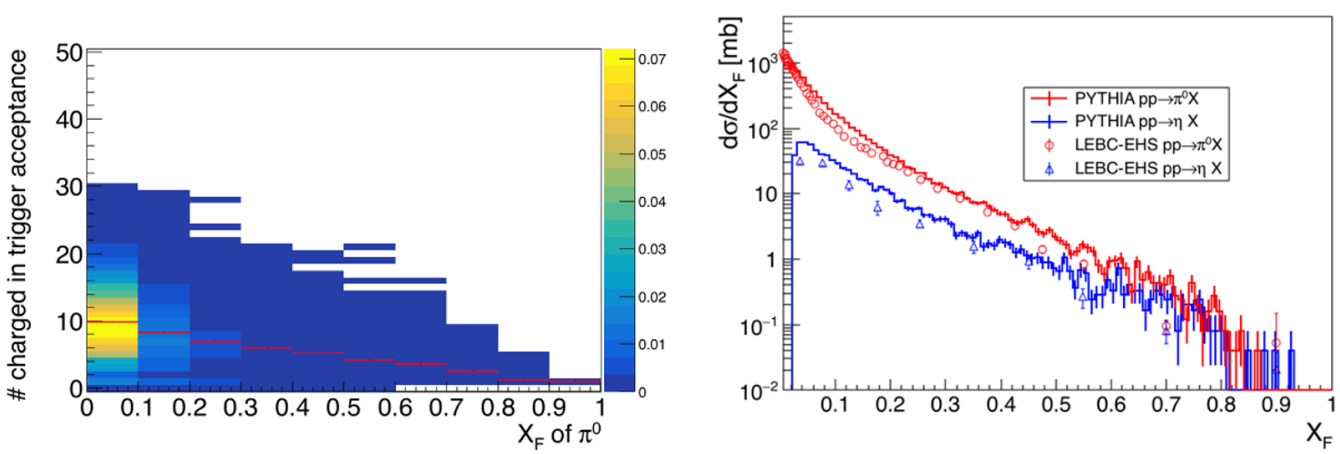

Figure 3. Left: number of charged particles in the acceptance of the trigger chambers in the LEBC-EHS experiment as a function of the Feynman $X_{F}$ of a co-emitted $\pi^{0}$ meson, as evaluated using a PYTHIA simulation: the red line indicates the average values for each $X_{F}$ bin. Right: data-MC comparison of the differential cross-section of neutral pions and $\eta$ mesons produced from $400 \mathrm{GeV}$ protons, after accounting for the trigger efficiency in the LEBC-EHS setup.

At $400 \mathrm{GeV}$ beam energy, the PYTHIA predicted yield is higher than the data especially at large $X_{F}$. To further scrutinize this issue, we first present the same comparison for charged pions at $400 \mathrm{GeV}$ beam energy: as shown in the right panel of figure 2, the data-MC comparison is significantly better than for $\pi^{0}$ mesons.

A careful review of [3] shows that a comprehensive data-MC comparison for neutral particles demands modelling and accounting for the trigger condition in the Monte Carlo output. In the LEBC-EHS data, the trigger requirement was to have 3 or more hits from charged particles in dedicated forward tracking stations right downstream of the hydrogen bubble-chamber target. As stated in [3], the trigger efficiency is as low as $2 \%$ when only two charged particles are emitted in acceptance and trigger inefficiencies may alter significantly the differential cross section at large $X_{F}$. We modelled this effect while analyzing the PYTHIA output ${ }^{1}$ and found that the number of charged particles emitted in the acceptance of the trigger chambers strongly depends on the $X_{F}$ of a co-emitted $\pi^{0}$, as shown in the left panel of figure 3. After correcting for the trigger efficiency, the differential cross section significantly changes, as shown in the right-hand side of figure 3 . The data-MC ratio within large- $X_{F}$ bins of sufficient statistics saturates at $\sim 0.8$, and one can safely use the entire $X_{F}$ range for sensitivity studies. The data-MC ratio for charged pions and $\eta$ mesons as a function of $X_{F}$ is higher than 0.8 for any value of $X_{F}$.

Checking the results of figure 10 in [1] for a number of benchmark points we verify that the sensitivity of proposed and past searches does not change very visibly in the double-log presentation such that the conclusions of [1] remain largely unchanged.

\section{Acknowledgments}

We are indebted to F. Kahlhoefer and his research group for their scrutiny in trying to reproduce our plots for the eta mesons, which made us spot the missing factor of 2 .

\footnotetext{
${ }^{1}$ The expected distribution of the interaction point has been considered as well.
} 
Open Access. This article is distributed under the terms of the Creative Commons Attribution License (CC-BY 4.0), which permits any use, distribution and reproduction in any medium, provided the original author(s) and source are credited.

\section{References}

[1] B. Döbrich, J. Jaeckel and T. Spadaro, Light in the beam dump. Axion-Like Particle production from decay photons in proton beam-dumps, JHEP 05 (2019) 213 [arXiv: 1904.02091] [INSPIRE].

[2] NA22 collaboration, Inclusive $\pi^{0}$ Production in $\pi^{+} p, K^{+} p$ and pp Interactions at 250-GeV/c, Z. Phys. C 35 (1987) 7 [inSPIRE].

[3] M. Aguilar-Benitez et al., Inclusive particle production in 400-GeV/c p p interactions, Z. Phys. C 50 (1991) 405 [INSPIRE]. 